Nota de investigación

\title{
Ensayo en invernadero de abonos verdes sobre las propiedades del suelo, producción de acelga e implicaciones ambientales
}

\author{
Montserrath Rojas-Velázquez ${ }^{1}$ \\ J. Carlos Rodríguez-Ortiz ${ }^{1 \S}$ \\ Jorge A. Alcalá-Jáuregui ${ }^{1}$ \\ Paola E. Díaz-Flores ${ }^{1}$ \\ Fernando J. Carballo-Méndez ${ }^{2}$ \\ Elizabeth Zúñiga Valenzuela ${ }^{3}$ \\ ${ }^{1}$ Facultad de Agronomía y Veterinaria-Universidad Autónoma de San Luis Potosí. Carretera Soledad de G. \\ S.-Matehuala km 14.5, San Luis Potosí, México. (montserojas1403@gmail.con; jorge.alcala@uaslp.mx; \\ paola.diaz@uaslp.mx). ${ }^{2}$ Facultad de Agronomía-Universidad Autónoma de Nuevo León. Hacienda el \\ Canadá, Escobedo, Nuevo León, México. (ing.fercarballo@ hotmail.com). ${ }^{3}$ Universidad Juárez del Estado \\ de Durango-Facultad de Agronomía y Zootecnia. Venecia, Gómez Palacio, Durango, México. CP. 35110. \\ (elizabeth.zunigaval@yahoo.com.mx). \\ §Autor para correspondencia: juancarlos.rodriguez@uaslp.mx.
}

\section{Resumen}

En este ensayo se estudió las leguminosas frijol dolichos (Lablab purpureos L. ex Sweet) y frijol yorimón (Vigna unguiculata L. Walp) como abonos verdes. Los objetivos del estudio fueron evaluar la capacidad de capturar carbono y fijar nitrógeno, así como medir los efectos de su incorporación en el suelo, la producción de acelga (Beta vulgaris var. cicla L.) y analizar las implicaciones ambientales. En los resultados L. purpureos produjo $1932 \mathrm{~kg}$ de materia seca en 90 días después de la siembra (869 $\mathrm{kg} \mathrm{ha}^{-1}$ de C) y fijó en sus tejidos $30 \mathrm{~kg}$ de $\mathrm{N} \mathrm{ha}^{-1}$, mientras que $V$. unguiculata produjo $2040 \mathrm{~kg}$ de materia seca en 80 días después de la siembra (918 $\mathrm{kg} \mathrm{ha}^{-1} \mathrm{de} \mathrm{C}$ ) y fijó en sus tejidos $40 \mathrm{~kg}$ de $\mathrm{N} \mathrm{ha}^{-1}$. Los efectos en el suelo no fueron significativos en ambos AV, en comparación con el tratamiento testigo ( $\sin$ AV). Sin embargo, ambos promovieron un incremento en peso de acelga ( $40 \%$ en L. purpureos y $31 \%$ en $V$. unguiculata). En lo ambiental, los abonos verdes pueden reducir la huella de carbono en $280 \mathrm{~kg} \mathrm{ha}^{-1}$ de $\mathrm{CO}_{2}$ equivalente al prescindir de fertilizantes químicos sintéticos nitrogenados en la producción de acelga. Los abonos verdes, como fuente de nutrientes, en especial N, podrían formar parte del manejo integral del suelo en los sistemas de producción hortícola de zonas semiáridas.

Palabras clave: Lablab purpureos L., Vigna unguiculata L., $\mathrm{CO}_{2}$, GEI, horticultura.

Recibido: abril de 2020

Aceptado: mayo de 2020 
Es fundamental producir alimentos de una manera que preserven el medio ambiente y la biodiversidad, implementando prácticas sostenibles que proporcionen alimentos saludables y nutritivos, servicios de los ecosistemas y resiliencia al cambio climático (Da Silva, 2018). Los sistemas convencionales de producción agrícola utilizan fertilizantes químicos sintéticos (FQS) para proporcionar a las plantas los nutrimentos necesarios. Sin embargo, tal práctica puede tener efectos colaterales negativos de contaminación y degradación del suelo, que se acentúan al combinar con la excesiva labranza de los suelos (García et al., 2010).

La utilización de abonos verdes (AV) forma parte de una estrategia tecnológica que ha sido probada por la investigación y la práctica de los agricultores en climas templados, mostrándose eficiente y económicamente viable y que tiene como objetivo el aumento y conservación de la materia orgánica del suelo y los nutrientes, especialmente el nitrógeno (N) (Kumar et al., 2013). Los incrementos del rendimiento asociados con el uso de cultivos de AV se han relacionado directamente con el contenido de nitrógeno de la cobertura y la materia seca total producida (Mehari et al., 2005).

Las propiedades físicas y químicas de los suelos se han utilizado para evaluar los efectos de la aplicación de diferentes fuentes de la materia orgánica en el suelo durante los experimentos a largo plazo (Tejada y González, 2005). Aunado a lo anterior, las aportaciones de nitrógeno al suelo, por los abonos verdes, significan un ahorro económico en nitrógeno de FQS, además de una reducción de gases de efecto de invernadero (GEI) (principalmente $\mathrm{N}_{2} \mathrm{O}$ ) que se emiten a la atmósfera en su fabricación y transporte.

Los AV son unas de las opciones más seguras para mejorar la fertilidad del suelo, pero es necesario evaluar diferentes especies de abonos en cada región a fin de seleccionar las más favorables (Beltrán et al., 2006a). Prager et al. (2012), mencionan que se requiere la réplica de ensayos en diversas zonas agroecológicas y la incorporación de herramientas metodológicas que permitan validar el carácter de los AV como tecnología multipropósito.

Debido a los escasos estudios sobre especies vegetales que puedan ser usadas como AV en zonas semiáridas, se realizó el presente estudio con el objetivo de evaluar la capacidad de las leguminosas dolichos (Lablab purpureos L. ex Sweet) y yorimón (Vigna unguiculata L. Walp) de capturar carbono y fijar nitrógeno, así como su efecto en algunas propiedades fisicoquímicas del suelo, en la producción de acelga (Beta vulgaris var. cicla L.) y analizar las implicaciones ambientales.

El ensayo se realizó en el Centro de Capacitación en Agricultura Protegida de la Universidad Autónoma de San Luis Potosí. Fue hecho en un ambiente protegido y utilizando macetas para tener un control de las variables de estudio. Los AV fueron sembrados en bolsas de polietileno color negro calibre 600 con capacidad de 12 L, que contenían suelo agrícola (migajón arcillo-arenoso). Cada maceta fue considerada como una repetición, en total fueron doce macetas por especie.

La humedad del suelo se mantuvo en un rango entre la capacidad de campo y $70 \%$ de humedad disponible en todo el ensayo. A partir de los cuarenta y cinco días y hasta la cosecha, se realizó una aplicación semanal de lixiviado de gallinaza diluido en el riego $(\mathrm{pH}$ y conductividad eléctrica de la solución orgánica fue de 6.5 y $1.3 \mathrm{dS} \mathrm{m}^{-1}$, respectivamente). Las AV fueron incorporadas al suelo en el inicio de la floración, a los 80 días después de la siembra (dds) para el frijol yorimón y a los 95 dds para el frijol dolichos. 
Previo a la incorporación, se colectaron cinco plantas de cada especie para los análisis de producción de biomasa y contenido de nitrógeno. El nitrógeno en planta se determinó por el método Kjeldhal y el carbono se estimó con base a la relación del peso seco con el carbono promedio en las plantas, que es de 45\% (Azcón y Talon, 2008). Con los datos de concentración nitrógeno y carbono por planta, se calcularon los valores por hectárea por medio de proporción directa, asumiendo una densidad de población de 60000 plantas ha $^{-1}$.

A los 30 días de la incorporación de los abonos verdes, se obtuvo una muestra compuesta de suelo para cada AV, la cual se utilizó para determinar la densidad aparente (método probeta), pH (potenciómetro, relación 1:2 suelo: agua), la conductividad eléctrica en pasta de saturación (conductímetro), materia orgánica (método de Walkley-Black) y nitrógeno total (microkjedhal) (Rodríguez y Rodríguez, 2011). Después de la incorporación de los AV, se realizó el ensayo con el cultivo subsecuente. Plántulas de acelga (Beta vulgaris var. cicla L.), de treinta y cinco días de edad y cuatro hojas verdaderas, fueron trasplantadas en las mismas bolsas.

Los tratamientos evaluados fueron: T1) suelo sin fertilización; T2) suelo con fertilizante químico sintético (una sola aplicación de $1 \mathrm{~g}$ por planta de triple 17, a los siete días después del trasplante); T3) suelo con residuos de dolichos; T4) suelo con residuos de frijol yorimón. Los tratamientos fueron establecidos bajo un diseño experimental completamente al azar con doce repeticiones (bolsas que ocuparon los AV). A partir de los seis días después del trasplante (ddt), y hasta la cosecha, se midieron las unidades SPAD cada semana con el equipo SPAD-502 Minolta Plus.

A los 72 días ddt se realizó la cosecha de las plantas de acelga, y se evaluaron las siguientes variables: a) clorofila (espectrofotometría); b) peso fresco de hojas y raíz; c) peso seco de hoja y raíz; d) altura de la planta; e) nitrógeno en tejido vegetal (método Kjeldhal). Los datos fueron sometidos a un análisis de varianza en el paquete estadístico Statistical Analysis System con la versión 9.0 (SAS Institute, 2002). En las variables donde se mostraron diferencias significativas se realizó una comparación de medias mediante la prueba de Tukey $(p<0.05)$.

Los resultados indican que los AV fueron estadísticamente iguales en cuanto a la producción de materia seca $(p<0.05)$. L . purpureos produjo $1932 \mathrm{~kg}$ de materia seca en 90 días después de la siembra (869 $\mathrm{kg} \mathrm{ha}^{-1}$ de C), mientras que $V$. unguiculata produjo $2040 \mathrm{~kg}$ de materia seca en 80 días después de la siembra $\left(918 \mathrm{~kg} \mathrm{ha}^{-1} \mathrm{de} \mathrm{C}\right)$. En cuanto al porcentaje de nitrógeno en tejido vegetal, ambos AV fueron estadísticamente iguales $(p<0.05)$, el promedio de $\mathrm{N}$ en tejido vegetal fue de $2 \%$ en planta completa.

La cantidad de nitrógeno contenido en la biomasa, y que puede ser incorporado al suelo, fue de 40 $\mathrm{kg} \mathrm{ha}^{-1}$ en frijol yorimón y $37 \mathrm{~kg} \mathrm{ha}^{-1}$ en frijol dolichos, obtenidos en 80 y 95 dds, respectivamente. El 75\% y $65 \%$ correspondería a la parte aérea de las plantas para yorimón y dolichos, respectivamente. Beltrán et al. (2009), reportó valores de 4 y 3.96\% de $\mathrm{N}$ en tejido de planta completa para dolichos y yorimón, respectivamente.

Beltrán et al. (2006b) mencionan que la incorporación de frijol dolichos como abono verde, en dosis de $6 \mathrm{t}$ de materia seca, tiene un aporte nitrogenado al suelo de $240 \mathrm{~kg} \mathrm{ha}^{-1}$ a los 160 días de establecida. García et al. (2010), mencionan que la descomposición del abono y la subsecuente liberación de $\mathrm{N}$ dependen, en mayor medida, de la calidad y cantidad de los residuos, la humedad y la temperatura del suelo, la mineralización y el $\mathrm{pH}$. 
Los efectos de los AV en el suelo no fueron estadísticamente diferentes en relación con el tratamiento testigo $(p<0.05)$. Sin embargo, los suelos con AV incrementaron $5 \%$ la densidad aparente, $11 \%$ la materia orgánica y 17\% nitrógeno inorgánico (Cuadro 1).

Cuadro 1. Efecto de los abonos verdes en las propiedades del suelo a los 30 días después de su incorporación.

\begin{tabular}{cccc}
\hline Propiedad del suelo & Suelo antes de abonos verdes & $\begin{array}{c}\text { Suelo con } \\
\text { dolichos }\end{array}$ & $\begin{array}{c}\text { Suelo con } \\
\text { yorimón }\end{array}$ \\
\hline Densidad $\left(\mathrm{g} \mathrm{cm}^{-3}\right)$ & $1.26 \pm 0.02^{*}$ & $1.2 \pm 0.02$ & $1.2 \pm 0.1$ \\
$\mathrm{pH}$ & $8 \pm 0.15$ & $8.1 \pm 0.25$ & $8 \pm 0.08$ \\
$\mathrm{CE}\left(\mathrm{dS} \mathrm{m}{ }^{-1}\right)$ & $1.1 \pm 0.17$ & $1 \pm 0.05$ & $1.1 \pm 0.15$ \\
Materia orgánica $(\%)$ & $0.7 \pm 0.1$ & $0.78 \pm 0.05$ & $0.76 \pm 0.03$ \\
Carbono orgánico $(\%)$ & $0.41 \pm 0.06$ & $0.45 \pm 0.03$ & $0.44 \pm 0.03$ \\
Nitrógeno inorgánico $(\mathrm{ppm})$ & $28.22 \pm 1.07$ & $32.5 \pm 0.09$ & $33 \pm 0.08$ \\
\hline
\end{tabular}

* $=$ los valores son las medias \pm error estándar.

Los efectos de los AV en las propiedades del suelo, en un primer ciclo de incorporación, han sido reportados por diversos autores. Beltrán et al. (2006b), encontraron que, en parcelas con incorporación de $\mathrm{AV}$, la materia orgánica se incrementó solo $0.17 \%$. Los cambios paulatinos en suelo por los AV pueden ser explicados por la estrecha relación $\mathrm{C} / \mathrm{N}$ de los $\mathrm{AV}$, que en este ensayo el promedio fue de 23 .

Dicho valor indica que los materiales pueden descomponerse rápidamente en el suelo, ser mineralizados (lo que aportaría nutrientes en el cultivo subsecuente) y aportar un carbono lábil (que es fuente de energía de los microorganismos del suelo, la gran mayoría benéficos para la agricultura) (Prager et al., 2012). Johnston et al. (2009), mencionan que el incremento en materia orgánica, durante el periodo de transición en el que se realizan aportaciones de AV ocurre lentamente y tarda varios años.

Por lo tanto, es posible que los efectos significativos en la productividad del suelo sean a largo plazo (Brechelt, 2004; García et al., 2010). Otros estudios han reportado cambios significativos en la materia orgánica desde el primer año, aunque la tasa de acumulación de materia orgánica es muy lenta (Herencia et al., 2008). Respecto a los efectos de los AV en el cultivo subsecuente, los resultados se aprecian en el Cuadro 2. Los AV promovieron significativamente un mayor peso fresco y seco en plantas de acelga $(p<0.05)$. Las unidades SPAD fueron iguales entre los cuatro tratamientos $(p<0.05)$ y la clorofila fue menor en las plantas a las que no se aplicó fertilizante ni AV (T1).

Para que un AV puede ser considerado como una fuente efectiva de nitrógeno para cultivos hortícolas, debe aportar suficiente $\mathrm{N}$ de acuerdo con los requerimientos del cultivo, y la liberación de $\mathrm{N}$ disponible debe ser sincronizada con la fenología de dicho cultivo (Hernández-Mendoza et al., 2007). Los resultados de este estudio permiten afirmar que los AV promovieron una mejor nutrición de nitrógeno que el tratamiento testigo con FQS (Cuadro 2). 
Cuadro 2. Resultados de las variables de estudio en acelga (Beta vulgaris var. cicla L.).

\begin{tabular}{ccccc}
\hline Variable & T1 sin fertilizante & T2 con FQS & T3 Dolichos & T4 Yorimón \\
\hline PFPA $(\mathrm{g})$ & $27.12 \pm 5.47 \mathrm{c}$ & $42.2 \pm 6.34 \mathrm{~b}$ & $61.22 \pm 3.81 \mathrm{a}$ & $59.02 \pm 8.45 \mathrm{a}$ \\
PFR $(\mathrm{g})$ & $8.78 \pm 2.16 \mathrm{c}$ & $23.62 \pm 3.01 \mathrm{~b}$ & $31.7 \pm 3.99 \mathrm{a}$ & $27.62 \pm 3.08 \mathrm{~b}$ \\
PFT $(\mathrm{g})$ & $35.9 \pm 6.59 \mathrm{c}$ & $65.82 \pm 6.68 \mathrm{~b}$ & $92.92 \pm 6.7 \mathrm{a}$ & $83.64 \pm 12.39 \mathrm{a}$ \\
PSPA (g) & $3.94 \pm 0.95 \mathrm{c}$ & $5.62 \pm 0.73 \mathrm{~b}$ & $7.94 \pm 1.56 \mathrm{a}$ & $7.54 \pm 1.04 \mathrm{a}$ \\
PSR (g) & $1.52 \pm 0.25 \mathrm{c}$ & $3.44 \pm 1 \mathrm{~b}$ & $4.52 \pm 1.17 \mathrm{a}$ & $4.34 \pm 1.36 \mathrm{a}$ \\
PST (g) & $5.46 \pm 1.03 \mathrm{c}$ & $9.06 \pm 1.14 \mathrm{~b}$ & $12.46 \pm 3.63 \mathrm{a}$ & $11.88 \pm 3.22 \mathrm{a}$ \\
Cl (mm/l) & $1.16 \pm 0.19 \mathrm{~b}$ & $1.52 \pm 0.09 \mathrm{ab}$ & $1.68 \pm 0.01 \mathrm{a}$ & $1.43 \pm 0.2 \mathrm{ab}$ \\
SPAD & $44.58 \pm 1.19 \mathrm{a}$ & $45.81 \pm 1.39 \mathrm{a}$ & $45.92 \pm 1.73 \mathrm{a}$ & $45.94 \pm 2.13 \mathrm{a}$ \\
$\mathrm{N}(\%)$ & $2.33 \pm 0.11 \mathrm{a}$ & $2.49 \pm 0.09 \mathrm{a}$ & $2.52 \pm 0.1 \mathrm{a}$ & $2.48 \pm 0.11 \mathrm{a}$ \\
\hline
\end{tabular}

Los valores son la media \pm error estándar. Medias con la misma letra en la misma fila no son significativamente diferente de acuerdo con la prueba de Tukey $(p<0.05),(\mathrm{n}=6)$. Fertilizante químico sintético (FQS); peso fresco parte aérea (PFPA); peso fresco raíz (PFR); peso fresco total (PFT); peso seco parte aérea (PSPA); peso seco raíz (PSR); peso seco total (PST); clorofila (Cl); unidades SPAD (SPAD); nitrógeno (N).

Las posibles causas del mayor rendimiento de las plantas de acelga, por efecto de los AV, son la diferencia de materia orgánica de los suelos por efecto de los AV (Cuadro 1), que fue 11\% más que los suelos sin AV (T1 y T2), la cual pudo haber afectado positivamente otros procesos del suelo: capacidad de intercambio catiónico, espacio poroso, retención de humedad, mayor actividad biológica, etc. (Martínez et al., 2008).

Aunado a lo anterior, el mayor contenido de nitrógeno inorgánico del suelo del tratamiento con frijol yorimón (17\%), aun cuando esta diferencia no se mostró en el contenido de nitrógeno en la planta, pudo haber contribuido con dicho aumento en el rendimiento. Singh et al. (2010), reportan que el frijol yorimón mostró un resultado similar a la aplicación de $30 \mathrm{~kg} \mathrm{ha}^{-1}$ de fertilizante nitrogenado, con lo que se logró incrementar la producción de materia seca de menta $23.4 \%$ y la producción de aceite esencial en $25.2 \%$.

En nuestro estudio, la aplicación de $1 \mathrm{~g} \mathrm{planta}^{-1}$ de fertilizante T17, es equivalente a $80 \mathrm{~kg}$ de $\mathrm{N}$ $\mathrm{ha}^{-1}$ (asumiendo una densidad de 80 mil plantas de acelga ha ${ }^{-1}$ ). Las implicaciones ambientales del uso de AV se consideran positivas en el sentido de no usar FQS, o reducirlos significativamente, como se demostró de manera particular en este ensayo. Los fertilizantes nitrogenados (como el nitrato de amonio y la urea), en su proceso de producción, tienen una huella de carbono de $3.5 \mathrm{~kg}$ de $\mathrm{CO}_{2}$ equivalente por cada $\mathrm{kg}$ de $\mathrm{N}$ producido (que es el caso de los fertilizantes fabricados en la unión europea).

Sin embargo, la huella de carbono puede ser mayor, como en el caso de los fertilizantes producidos en china, donde la huella de carbono puede ser mayor a $10 \mathrm{~kg}$ de $\mathrm{CO}_{2}$ equivalente (Bentrup, 2016). De esta manera, el prescindir de $80 \mathrm{~kg}_{\mathrm{ge} \mathrm{N} \mathrm{ha}}{ }^{-1}$ de fertilizante nitrogenado (que se aplicó en forma de triple 17 en el tratamiento testigo para la producción de acelga), se estaría evitando, al menos,

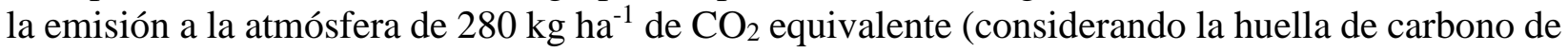
$3.5 \mathrm{~kg} \mathrm{CO} 2 \mathrm{eq})$. 
Lo anterior es significativo, si consideramos que la mayoría de los cultivos de importancia económica, tienen requerimientos de nutrientes desde $150 \mathrm{~kg} \mathrm{de} \mathrm{N} \mathrm{ha}^{-1}$ en el caso de trigo y hasta más de $1000 \mathrm{~kg}_{\text {de }} \mathrm{N} \mathrm{ha}^{-1}$ en tomate de invernadero, lo que representarían 540 y $3600 \mathrm{~kg} \mathrm{ha}^{-1} \mathrm{de}$ $\mathrm{CO}_{2}$ equivalente, respectivamente. La FAO (2014), señala que los gases de efecto invernadero se han duplicado en las actividades agrícolas en Latinoamérica en los últimos cincuenta años, por lo que es fundamental el fomento a la absorción y retención de dichos gases.

Otra implicación relevante, es la incorporación de carbono al suelo (en este ensayo $V$. unguiculata capturó $918 \mathrm{~kg} \mathrm{ha}^{-1} \mathrm{de} \mathrm{C}$ ), donde una parte puede pasar a ser parte del carbono estable del suelo. Reicosky (2002), menciona que, a nivel mundial, las pérdidas de carbono orgánico de los suelos agrícolas fluctúan entre 30 y $50 \%$ de su nivel inicial y que pocas prácticas se realizan intencionalmente para su conservación dentro de la agricultura convencional.

Por su parte, Lal (2004), menciona que 58\% de las emisiones de $\mathrm{CO}_{2}$ emitido a la atmósfera es atribuido a la pérdida de materia orgánica provocado por las labores de labranza primarias y técnicas de cultivo convencional. Lal (2011), señala que los suelos agrícolas pueden ser importante sumideros de carbono si se lleva a cabo un uso correcto.

\section{Conclusiones}

Las leguminosas dolichos (Lablab purpureos) y yorimón (Vigna unguiculata L.) no mejoraron significativamente las propiedades físicas y químicas del suelo. Sin embargo, promovieron un mayor rendimiento de acelga en relación con la producción con fertilizante químico sintético (40\% en $L$. purpureos y $31 \%$ en $V$. unguiculata). La utilización de los abonos verdes contribuye a suplir la utilización de fertilizantes químico sintético en dicha hortaliza y, en el caso de los nitrogenados, a disminuir la huella de carbono que se genera por su fabricación.

\section{Literatura citada}

Azcón, B. J. y Talon, M. 2008. Fundamentos de la fisiología vegetal. $2^{\text {nd }}$ Edición. Ed. McGrawHill. $631 \mathrm{p}$.

Beltrán-Morales, F. A.; García-Hernández, J. L.; Zamora-Salgado, S.; Fenech-Larios, L. y MurilloMarcial, R. 2006a. El uso de abonos verdes en la agricultura orgánica. 159-205 pp.

Beltrán-Morales, F. A.; García-Hernández, J. L.; Valdez-Cepeda, R. D.; Murillo-Amador, B.; Troyo-Diéguez, E.; Larrinaga, J. y Beltrán-Morales, L. F. 2006b. Efecto de sistemas de labranza e incorporación de abono verde (Lablab purpureus L.) sobre la respiración edáfica en un yermosol háplico. Interciencia. 31:226-230.

Beltrán-Morales, F. A.; García-Hernández, J. L.; Ruiz-Espinoza, F. H.; Fenech-Larios, L.; MurilloAmador, B.; Palacios, A y Troyo-Diéguez, E. 2009. Nutrimental potential of red dolichos, brown dolichos and cowpea for green manure produced under three tillage Systems. Trop. Subtrop. Agroecosystems. 10:487-49.

Bentrup, F.; Hoxha, A. and Christiensen, B. 2016. Carbon footprint analysis of mineral fertilizer production in Europe and other world regions. In: Conference: the $10^{\text {th }}$ International Conference on Life Cycle Assessment of Food (LCA Food 2016). At: University College Dublin (UCD), Dublin, Ireland. 
Brechelt, A. 2004. El manejo ecológico del suelo. Fundación Agricultura y Medio Ambiente (FAMA). Red de acción en plaguicidas y sus alternativas para América Latina (RAP-AL) (Ed.). Santiago de Chile, Chile.

Da Silva, J. G. 2018. Time to embrace sustainable agriculture-FAO. https://www.standarmedia. co.ke/farmers/article/2001297618/time-to-embrace-sustainable-agriculture-fao.

FAO. 2014. Latinoamérica duplicó sus emisiones agrícolas de gases de efecto invernadero en los últimos 50 años. http://www.fao.org/ americas/noticias/ver/es/c/240450/.

García-Hernández, J. L.; Murillo-Amador, B.; Nieto-Garibay, A.; Fortis-Hernández, M.; MárquezHernández, C.; Castellanos-Pérez, E.; Quiñones-Vera, J. J. y Ávila-Serrano, N. Y. 2010. Avances en investigación y perspectivas del aprovechamiento de los abonos verdes en la agricultura. Terra Latinoam. 18(4):391-399.

Herencia, J.; Ruiz, J.; Melero, S.; García, P. and Maqueda, C. 2008. A short-term comparison of organic v. conventional agriculture in a silty loam soil using two organic amendments. The Journal of Agricultural Science. 146(6):677-687. Doi: 10.1017/S0021859608008071.

Hernández-Mendoza, T. M.; Salcedo-Pérez, E.; Arévalo-Galarza, G. y Galvis-Spinola, A. 2007. Evaluación de la concentración de lignina como indicador de la capacidad de aporte de nitrógeno de residuos orgánicos. Rev. Chapingo Ser. Cienc. Fores. Amb. 13:5-13.

Johnston, A. E.; Poulton, P. R. and Coleman, K. 2009. Soil organic matter: its importance in sustainable agriculture and carbon dioxide fluxes. Adv. Agron. 101:1-57.

Kumar, A.; Kumar, G. D. and Mukesh, K. 2013. Green manure crops: A boon for agricultural soil. International J. Agr. Environ. Biotech. 6:193-198.

Lal, R. 2004. Soil carbon sequestration impacts on global climate change and food security. Science. 304(5677):1623-1627.

Lal, R. M 2011. Sequestering carbon in soils of agro-ecosystems. Food Policy. 36(1):S33-S39.

Martínez, E. H.; Fuentes, E. J. P. y Acevedo, E. 2008. Carbono orgánico y propiedades del suelo. J. Soil Sc. Plant Nutr. 8(1):68-96.

Mehari, A. F.; Mrema, A. and Weih, M. 2005. The suitability of Acacia tortilis as an alternative tree manure crop to Leucaena leucocephala in sub-Saharan Africa. Afr. J. Ecol. 43(2):162-165.

Prager-Mosquera, M.; Sanclemente-Reyes, O.; Sanchez-de-Prager, M.; Miller-Gallego, J. y ÁngelSánchez, D. 2012. Abonos verdes: tecnología para el manejo agroecológico de los cultivos. Agroecología. 7:53-62.

Reicosky, D. C. 2002. Tillage and gas exchange. In: Lal, R. (Ed.). Encyclopedia of soil science. Boca Raton, FL: Taylor \& Francis. 1333-1335 p.

Rodríguez, X. y Rodríguez X. 2011. Métodos de análisis de suelos y plantas: criterios de interpretación. 2a (Ed.). Trillas, México, DF. 239 p.

SAS Institute. 2002. SAS/STAT user's guide, version 5.0. $4^{\text {th }}$ (Ed.) SAS Inst., Cary, NC.

Singh, M.; Singh, A.; Singh, S.; Tripathi, R. S.; Singh, A. K. and Patra, D. D. 2010. Cowpea (Vigna unguiculata L. Walp.) as a green manure to improve the productivity of a menthol mint (Mentha arvensis L.) intercropping system. Indust. Crops Prod. 31:289-293.

Tejada, M. and González, J. L. 2005. Beet vinasse applied to wheat under dryland conditions affects soil properties and yield. Eur. J. Agron. 23:336-347. 